Hua-Rui Wang*

\title{
Crystal structure of poly[diaqua-( $\mu_{3}$-fumarato)- ( $\mu_{3}$-maleato)- $\left(\mu_{4}-1,2,4,5\right.$-tetrakis $((1 H-1,2,4-$ triazol-1-yl)methyl)benzene)tetracadmium(II)] dihydrate, $\mathrm{C}_{34} \mathrm{H}_{32} \mathrm{~N}_{12} \mathrm{O}_{9} \mathrm{Cd}_{4}$
}

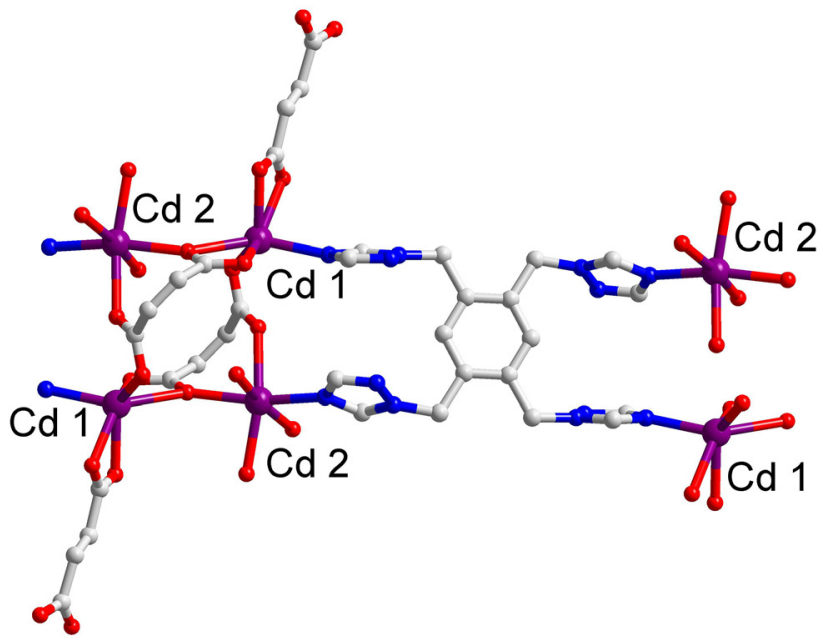

https://doi.org/10.1515/ncrs-2021-0475

Received December 15, 2021; accepted February 3, 2022; published online February 18, 2022

\section{Abstract}

$\mathrm{C}_{34} \mathrm{H}_{32} \mathrm{~N}_{12} \mathrm{O}_{9} \mathrm{Cd}_{4}$, monoclinic, $P \overline{1}$ (no. 2), $a=9.894(2) \AA$, $b=9.942(2) \AA, c=10.896(2) \AA, \alpha=94.35(3)^{\circ}, \beta=96.80(3)^{\circ}$, $y=93.35(3)^{\circ}, V=1058.7(4) \AA^{3}, Z=1, R_{g t}(F)=0.0406$, $w R_{\text {ref }}\left(F^{2}\right)=0.0984, \mathrm{~T}=293(2) \mathrm{K}$.

\section{CCDC no.: 2149837}

A part of the title coordination polymer is shown in the figure. Tables 1 and 2 contain details on crystal structure and measurement conditions and a list of the atoms including atomic coordinates and displacement parameters.

\section{Source of material}

Maleic acid (0.012 g, $0.1 \mathrm{mmol})$, 1,2,4,5-tetrakis(1,2,4-triazol1-ylmethyl)benzene (0.02 g, $0.05 \mathrm{mmol})$ and cadmium(II)

*Corresponding author: Hua-Rui Wang, College of Chemistry and Chemical Engineering, and Henan Key Laboratory of FunctionOriented Porous Materials, LuoYang Normal University, Luoyang, Henan 471934, P. R. China, E-mail: wanghrly@126.com. https://orcid. org/0000-0003-4069-3350
Table 1: Data collection and handling.
Crystal:

Size:

Wavelength:

Diffractometer, scan mode:

$\theta_{\text {max }}$, completeness:

$N(h k l)_{\text {measured }}, N(h k l)_{\text {unique }}, R_{\text {int }}$ :

Criterion for $l_{\mathrm{obs}}, N\left(h k l_{\mathrm{gt}}\right.$ :

$N(\text { param })_{\text {refined: }}$

Programs:
Colorless block

$0.26 \times 0.21 \times 0.15 \mathrm{~mm}$

Mo $K \alpha$ radiation $(0.71073 \AA)$

$2.08 \mathrm{~mm}^{-1}$

Rigaku Saturn 724, $\omega$

$27.9^{\circ}, 99 \%$

$12,985,4985,0.025$

$I_{\text {obs }}>2 \sigma\left(I_{\text {obs }}\right), 4319$

316

CrysAlis $^{\text {PRO }}[1]$, Olex2 [2], SHELX [3, 4] acetate $(0.023 \mathrm{~g}, 0.1 \mathrm{mmol})$ were added to the mixture of acetonitrile $(7 \mathrm{~mL})$ and water $(1 \mathrm{~mL})$ in a Teflon-lined stainless steel reactor. The mixture was heated at $433 \mathrm{~K}$ for three days, and then slowly cooled down to room temperature. Colorless crystals of the title compound were obtained.

\section{Experimental details}

The crystallographic data of title complex was collected on a Rigaku Saturn 724 CCD diffractometer at room temperature. Absorption corrections were applied by using multiscan program [1]. Using Olex2 [2], the structure was solved with the ShelXT [3] structure solution program using Intrinsic Phasing and refined with the ShelXL [4] refinement package. The $\mathrm{H}$ atoms bonded to $\mathrm{C}$ atoms were fixed, with $\mathrm{C}-\mathrm{H}$ distance of $0.93 \AA$ Å; and/or positioned geometrically in the riding-model approximation, with $\mathrm{C}-\mathrm{H}$ distance of $0.97 \AA$; $U_{\text {iso }}(\mathrm{H})=1.2 U_{\text {eq }}(\mathrm{C}), U_{\text {iso }}(\mathrm{H})=1.5 U_{\text {eq }}(\mathrm{O})$.

\section{Comment}

A serial of complexes based on flexible 1,2,4-triazole-containing ligands were reported, as 1,4-bis(1,2,4-triazol-1-ylmethyl)benzene (bbtz), 1,4-bis(1,2,4-triazol-1-yl)butane (btb), 
Table 2: Fractional atomic coordinates and isotropic or equivalent isotropic displacement parameters $\left(\AA^{2}\right)$.

\begin{tabular}{|c|c|c|c|c|}
\hline Atom & $x$ & $y$ & $z$ & $U_{\text {iso }} * / U_{\text {eq }}$ \\
\hline Cd1 & $0.38499(3)$ & $0.60679(3)$ & $0.26674(3)$ & $0.04006(10)$ \\
\hline $\mathrm{Cd} 2$ & $0.57500(3)$ & $0.82836(3)$ & $-0.01463(3)$ & $0.04661(11)$ \\
\hline 01 & $0.4111(3)$ & $0.7063(4)$ & 0.0711 (3) & $0.0540(8)$ \\
\hline 02 & $0.2142(4)$ & $0.6124(5)$ & 0.0908 (3) & $0.0824(14)$ \\
\hline 03 & $0.4093(3)$ & $0.4703(3)$ & $-0.2634(3)$ & $0.0474(7)$ \\
\hline 04 & $0.5338(3)$ & $0.6431(3)$ & $-0.1625(3)$ & $0.0538(8)$ \\
\hline 05 & $0.3158(4)$ & $0.8051(4)$ & $0.3513(3)$ & $0.0603(9)$ \\
\hline 06 & 0.4559 (4) & $0.7031(4)$ & 0.4779 (3) & $0.0587(9)$ \\
\hline 07 & $0.3467(5)$ & $1.1137(4)$ & $0.7466(4)$ & $0.0935(16)$ \\
\hline 08 & $0.4597(4)$ & 0.9954 (3) & $0.8763(3)$ & $0.0518(8)$ \\
\hline 012 & $0.7248(4)$ & 0.6799 (4) & 0.0854 (4) & $0.0772(12)$ \\
\hline $\mathrm{H} 12 \mathrm{~A}$ & 0.7420 & 0.7300 & 0.1529 & $0.116^{\star}$ \\
\hline H12B & 0.6860 & 0.6208 & 0.1298 & $0.116^{\star}$ \\
\hline N1 & $-0.2320(3)$ & $-0.0992(3)$ & $0.9098(3)$ & $0.0400(8)$ \\
\hline N2 & $-0.0813(3)$ & $0.0178(3)$ & $0.8250(3)$ & $0.0328(7)$ \\
\hline N3 & $-0.0151(4)$ & $-0.0875(4)$ & $0.8704(3)$ & $0.0431(8)$ \\
\hline N4 & $0.2324(4)$ & $0.4668(4)$ & $0.3446(3)$ & $0.0410(8)$ \\
\hline N5 & $0.0310(4)$ & $0.3847(4)$ & $0.3897(4)$ & $0.0471(9)$ \\
\hline N6 & $0.1342(3)$ & $0.3540(3)$ & $0.4759(3)$ & $0.0370(7)$ \\
\hline $\mathrm{C} 1$ & $0.4092(5)$ & $1.0149(5)$ & $0.7678(4)$ & $0.0476(10)$ \\
\hline $\mathrm{C} 4$ & $0.3644(6)$ & $0.9042(5)$ & $0.5557(4)$ & $0.0584(13)$ \\
\hline $\mathrm{H} 4$ & 0.3010 & 0.9678 & 0.5384 & $0.070^{\star}$ \\
\hline C5 & $0.4334(5)$ & $0.9124(5)$ & $0.6660(4)$ & $0.0480(10)$ \\
\hline H5 & 0.5004 & 0.8522 & 0.6821 & $0.058^{\star}$ \\
\hline C6 & $0.2374(4)$ & $0.6923(5)$ & $-0.1023(4)$ & $0.0471(10)$ \\
\hline H6 & 0.1568 & 0.7361 & -0.1127 & $0.056^{\star}$ \\
\hline $\mathrm{C} 7$ & $0.1074(5)$ & $0.2752(4)$ & $0.5791(4)$ & $0.0461(10)$ \\
\hline $\mathrm{H} 7 \mathrm{~A}$ & 0.0433 & 0.3205 & 0.6259 & $0.055^{\star}$ \\
\hline H7B & 0.1917 & 0.2719 & 0.6339 & $0.055^{\star}$ \\
\hline C8 & $-0.1089(4)$ & $-0.1541(4)$ & $0.9205(4)$ & $0.0419(9)$ \\
\hline H8 & -0.0930 & -0.2324 & 0.9600 & $0.050^{*}$ \\
\hline C10 & $-0.0157(4)$ & $0.1127(4)$ & $0.7521(3)$ & $0.0370(8)$ \\
\hline $\mathrm{H} 10 \mathrm{~A}$ & 0.0758 & 0.1389 & 0.7924 & $0.044^{\star}$ \\
\hline $\mathrm{H} 10 \mathrm{~B}$ & -0.0662 & 0.1934 & 0.7494 & $0.044^{\star}$ \\
\hline C11 & $0.0576(4)$ & 0.0764 (4) & $0.4190(3)$ & $0.0353(8)$ \\
\hline H11 & 0.0974 & 0.1286 & 0.3638 & $0.042^{\star}$ \\
\hline C12 & $0.0959(4)$ & $0.4513(4)$ & $0.3138(4)$ & $0.0416(9)$ \\
\hline $\mathrm{H} 12$ & 0.0517 & 0.4855 & 0.2438 & 0.050 * \\
\hline $\mathrm{C} 23$ & $0.0503(4)$ & $0.1323(4)$ & $0.5380(3)$ & $0.0331(8)$ \\
\hline C24 & $-0.0077(4)$ & $0.0545(4)$ & $0.6208(3)$ & $0.0312(7)$ \\
\hline $\mathrm{C} 25$ & $0.2921(4)$ & $0.6688(4)$ & $0.0268(4)$ & $0.0399(9)$ \\
\hline $\mathrm{C} 26$ & $-0.2103(4)$ & $0.0081(4)$ & $0.8486(3)$ & $0.0347(8)$ \\
\hline $\mathrm{H} 26$ & -0.2755 & 0.0681 & 0.8254 & $0.042^{\star}$ \\
\hline $\mathrm{C} 27$ & $0.4213(4)$ & $0.5867(4)$ & $-0.2082(3)$ & $0.0391(9)$ \\
\hline C29 & $0.2924(5)$ & $0.6568(5)$ & $-0.2033(4)$ & $0.0463(10)$ \\
\hline H29 & 0.2467 & 0.6774 & -0.2784 & $0.056^{\star}$ \\
\hline C30 & $0.3822(5)$ & $0.7974(5)$ & $0.4556(4)$ & $0.0455(10)$ \\
\hline C31 & $0.2518(4)$ & $0.4028(4)$ & $0.4478(4)$ & $0.0416(9)$ \\
\hline H31 & 0.3361 & 0.3940 & 0.4931 & 0.050 * \\
\hline $09^{\mathrm{a}}$ & 0.0447 (13) & $0.4372(13)$ & $-0.0431(14)$ & $0.148(5)$ \\
\hline$H 9 A^{a}$ & -0.0326 & 0.3931 & -0.0537 & $0.222^{\star}$ \\
\hline$H 9 B^{a}$ & 0.0959 & 0.3873 & -0.0812 & $0.222^{\star}$ \\
\hline
\end{tabular}

accupancy: 0.5 . 1,3,5-tris(1,2,4-triazol-1-ylmethyl)-2,4,6-trimethylbenzene (tttmb) [5-12]. To further understand the coordination principle for these coordination complexes, we selected the flexible tetrapodal 1,2,4,5-tetra(1,2,4-triazol-1-ylmethyl)benzene $(t t y b)$ as a functional ligand and used the "mixligand" synthetic strategy to explore the assembly with "mixed" ttyb and aromatic polycarboxylate ligands [13-15]. In this context, we reacted 1,2,4,5-tetra(1,2,4-triazol-1-ylmethyl)benzene $(t t y b)$ with $\mathrm{Cd}^{2+}$ ions, then we added maleic acid $\left(\mathrm{H}_{2} \mathrm{mal}\right)$ as a precursor of an auxiliary ligand.

The asymmetric unit of compound 1 consists of two crystallographically independent $\mathrm{Cd}(\mathrm{II})$ atoms, one half of a tty $b$ ligand, one maleate ligand ( $m a l$ ), one fumarate (fum) ligand and two water molecules. The Cd1 is six-coordinated by three carboxylate oxygen atoms from two fum ligands, two carboxylate oxygen atoms from one mal ligand and one nitrogen atom from one ttyb ligand. This results in a distorted octahedral coordination of $\mathrm{Cd} 1$. The $\mathrm{Cd} 2$ is also six-coordinated by two carboxylate oxygen atoms from one fum ligand, two carboxylate oxygen atoms from two mal ligands, one nitrogen atom from tty $b$ ligand and one oxygen atom from coordinated water molecule. The $\mathrm{Cd}-\mathrm{O}$ bond lengths are in the range of 2.220(3)-2.451(3) $\AA$. The Cd-N lengths are 2.275(3) $\AA$ and 2.268(3) $\AA$. Moreover, in malate dianion, carboxylate groups with two kinds of coordination modes have been found; one is bridging and the other chelating-bridging. In fumarate dianion, carboxylate groups with two chelating-bridging coordination modes have been found. The fum ligand coordinates to the three $\mathrm{Cd}$ center in chelating mode. Each malate dianion ligand coordinates to three $\mathrm{Cd} 1$ and $\mathrm{Cd} 2$ atoms to generate a 2D layer. tty $b$ acts as a $\mu 4$-bridge linking four $\mathrm{Cd}(\mathrm{II})$ ions, and connect the 2D layer to extend to a 3D network. $\mathrm{H}_{2}$ male was used as initial reactant, but the mal and fum ligands were found in the resultant crystal structure. For the male ligand, the conformation transformation happened in situ hydrothermal reaction process $[16,17]$.

Author contributions: The author has accepted responsibility for the entire content of this submitted manuscript and approved submission.

Research funding: This work was supported by Key Scientific Research Projects of Higher Education of Henan Province (16A150016, DOI: 10.13039/501100013066) and Henan Province Natural Science Foundation (182300410237, DOI: 10.13039/501100006407).

Conflict of interest statement: The author declares no conflicts of interest regarding this article. 


\section{References}

1. Oxford Diffraction Ltd. CrysAlis ${ }^{\mathrm{PRO}}$ : Abingdon, Oxfordshire, England, 2006.

2. Dolomanov O. V., Bourhis L. J., Gildea R. J., Howard J. A. K., Puschmann H. OLEX2: a complete structure solution, refinement and analysis program. J. Appl. Crystallogr. 2009, 42, 339-341.

3. Sheldrick G. M. SHELXTL - integrated space-group and crystalstructure determination. Acta Crystallogr. 2015, A71, 3-8.

4. Sheldrick G. M. Crystal structure refinement with SHELXL. Acta Crystallogr. 2015, C71, 3-8.

5. Chang X.-H., Yang X.-G., Zhai Z.-M., Chen J.-Y., Li F.-F. Synthesis, structure and highly enhanced phosphorescence of a cadmium(II) coordination polymer assembled with 1,4-naphthalenedicarboxylic acid and 2-propylimidazole. Chin. J. Struct. Chem. 2021, 40, 187-192.

6. Liu X., Du L., Li R., Ma N., You M., Feng X. Different effects in the selective detection of aniline and $\mathrm{Fe}^{3+}$ by lanthanide-based coordination polymers containing multiple reactive sites. CrystEngComm 2020, 22, 2837-2844.

7. Yang X. G., Zhai Z. M., Lu X. M., Zhao Y., Chang X. H., Ma L. F. Room temperature phosphorescence of $\mathrm{Mn}$ (II) and $\mathrm{Zn}$ (II) coordination polymers for photoelectron response applications. Dalton Trans. 2019, 48, 10785-10789.

8. Lin Z.-J., Lü J., Hong M., Cao R. Metal-organic frameworks based on flexible ligands (FL-MOFs): structures and applications. Chem. Soc. Rev. 2014, 43, 5867-5895.

9. Qin J.-H., Xu P., Huang Y.-D., Xiao L.-Y., Lu W., Yang X.-G., Ma L., Zang S.-Q. High loading of $\mathrm{Mn}$ (II)-metalated porphyrin in MOF for photocatalytic $\mathrm{CO}_{2}$ reduction in gas-solid condition. Chem. Commun. 2021, 57, 8468-8471.
10. Hawes C. S., Hamilton S. E., Hicks J., Knowles G. P., Chaffee A. L., Turner D. R., Batten S. R. Coordination chemistry and structural dynamics of a long and flexible piperazine-derived ligand. Inorg. Chem. 2016, 55, 6692-6702.

11. Miao S.-B., Sun X.-J., Wang K.-X., Xu C.-Y., Li Z.-H., Wang Z.-Q. Effect of charge on the structures of $\mathrm{Zn}$ (II) coordination polymers with triazole-carboxylate ligands: syntheses, structures, and luminescent properties. Crystals 2018, 8, 312.

12. Qin J. H., Zhang H., Sun P., Huang Y. D., Shen Q., Yang X. G., Ma L. F. Ionic liquid induced highly dense assembly of porphyrin in MOF nanosheets for photodynamic therapy. Dalton Trans. 2020, 49, 17772-17778.

13. Xu G.-C., Hua Q., Okamura T., Bai Z.-S., Ding Y.-J., Huang Y.-Q., Liu G.-X., Sun W.-Y., Ueyama N. Cadmium(II) coordination polymers with flexible tetradentate ligand 1,2,4,5-tetrakis(imidazol1-ylmethyl)benzene: anion effect and reversible anion exchange property. CrystEngComm 2009, 11, 261-270.

14. Hua Q., Zhao Y., Xu G.-C., Chen M.-S., Su Z., Cai K., Sun W.-Y. Synthesis, structures, and properties of zinc(II) and cadmium(II) complexes with 1,2,4,5-tetrakis(imidazol-1-ylmethyl)benzene and multicarboxylate ligands. Cryst. Growth Des. 2010, 10, 2553-2562.

15. Hua Q., Su Z., Zhao Y., Okamura T., Xu G. C., Sun W.-Y., Ueyama N. Synthesis, structure and property of manganese(II) complexes with mixed tetradentate imidazole-containing ligand and benzenedicarboxylate. Inorg. Chim. Acta 2010, 363, 3550-3557.

16. Zhang S., Huang L., Qu L., Peng H., Zhao Y. Two novel R- and $\mathrm{S}$-malato-bridged coordination polymers by reacting lanthanide chloride and maleic anhydride, 1,10-phenanthroline at hydrothermal condition. J. Mol. Struct. 2006, 787, 14-17.

17. Bai H., Ma J., Liu Y., Yang J. Hydrothermal syntheses, crystal structures and topological analyses of four new 3D coordination polymers with mixed ligands. Inorg. Chim. Acta 2011, 376, 332-339. 